\title{
Existence of Standing Waves for Dirac Fields with Singular Nonlinearities
}

\author{
Mikhael Balabane ${ }^{1,2}$, Thierry Cazenave $^{3}$ and Luis Vázquez ${ }^{4}$ \\ 1 Département de Mathématiques, Université de Reims-Champagne-Ardennes, B.P. 347, F-51062 \\ Reims Cedex, France \\ 2 CMA, École Normale Supérieure, 45, rue d'Ulm, F-75252 Paris Cedex 05, France \\ 3 Analyse Numérique, Université Pierre et Marie Curie, 4, place Jussieu, F-75252 Paris Cedex \\ 05, France \\ 4 Departamento de Fisica Teorica, Facultad de Ciencias Fisicas, Universidad Complutense, \\ E-28040 Madrid, Spain
}

Received July 10, 1989; in revised form February 2, 1990

Abstract. We prove the existence of stationary states for nonlinear Dirac equations of the form

$$
i \sum_{\mu=0}^{3} \gamma^{\mu} \partial_{\mu} \psi-M \psi+F(\bar{\psi} \psi) \psi=0,
$$

where $M>0$ and $F$ is a singular self-interaction. In particular, in the model case where $F(s)=-s^{-\alpha}$, for some $0<\alpha<1$, and for every $\omega>M$, there exists a solution of (E) of the form $\psi(t, x)=e^{i \omega t} \varphi(x)$, where $x_{0}=t$ and $x=\left(x_{1}, x_{2}, x_{3}\right)$, such that $\varphi$ has compact support. If $0<\alpha<1 / 3$, then $\varphi$ is of class $C^{1}$. If $1 / 3<\alpha<1$, then $\varphi$ is continuously differentiable, except on some sphere $\{|x|=R\}$, where $|\nabla \varphi|$ is infinite.

\section{Introduction}

In this paper, we study the existence of stationary states for nonlinear Dirac equations of the form

$$
i \sum_{\mu=0}^{3} \gamma^{\mu} \partial_{\mu} \psi-M \psi+F(\bar{\psi} \psi) \psi=0
$$

We consider here the case where $F$ is a singular self-interaction.

The notation is the following. $\psi: \mathbf{R}^{4} \rightarrow \mathbf{C}^{4}, \partial_{\mu}=\partial / \partial x_{\mu}, M$ is a positive constant, $\bar{\psi} \psi=\left(\gamma^{0} \psi, \psi\right)$, where $(\cdot, \cdot)$ is the usual scalar product in $\mathbf{C}^{4}$ and the $\gamma^{\mu \text { 's }}$ are the $4 \times 4$ matrices of the Pauli-Dirac representation (see $[14,15,17,18]$ ), given by

$$
\gamma^{0}=\left(\begin{array}{cc}
I & 0 \\
0 & -I
\end{array}\right) \text { and } \gamma^{\mu}=\left(\begin{array}{cc}
0 & \sigma^{k} \\
-\sigma^{k} & 0
\end{array}\right), \text { for } k=1,2,3 \text {, }
$$


where

$$
\sigma^{1}=\left(\begin{array}{ll}
0 & 1 \\
1 & 0
\end{array}\right), \quad \sigma^{2}=\left(\begin{array}{cc}
0 & -i \\
i & 0
\end{array}\right), \quad \sigma^{3}=\left(\begin{array}{cc}
1 & 0 \\
0 & -1
\end{array}\right) .
$$

Finally, $F: \mathbf{R} \rightarrow \mathbf{R}$ models the nonlinear interaction.

We are interested in standing waves (or stationary states, or localized solutions) of (1.1). In other words, we look for solutions $\psi$ of the form

$$
\psi(t, x)=e^{i \omega t} \varphi(x)
$$

where $x_{0}=t$ and $x=\left(x_{1}, x_{2}, x_{3}\right)$. In addition we seek finite energy solutions; and so we want $\varphi$ to be at least integrable. The equation for $\varphi: \mathbf{R}^{3} \rightarrow \mathbf{C}^{4}$ is

$$
i \sum_{k=1}^{3} \gamma^{k} \partial_{k} \varphi-M \varphi+\omega \gamma^{0} \varphi+F(\bar{\varphi} \varphi) \varphi=0 .
$$

Nonlinear spinor fields giving rise to equations of the form (1.2) with smooth nonlinearities were considered first Ivanenko [8], Weyl [19], Heisenberg [7] and Finkelstein, Fronsdal and Kaus [6]. Later, Soler [10] proposed the scalar fourth order self-coupling as a model of extended fermions. A summary of such models is found in Rañada [13]. The mathematical study of (1.2) in the non-singular case was initiated by Vázquez [17], who gave necessary conditions for the existence of nontrivial solutions. Then Cazenave and Vázquez [4] established the existence of solutions under certain hypotheses on $F$. Later, Merle [12] generalized that result to a wider class of nonlinearities. Then, Balabane, Cazenave. Douady and Merle [2] established the existence of infinitely many solutions to (1.2) (see also Balabane [1] and Cazenave [3]). These results apply to the model case $F(s)=|s|^{p-1}$, for $p>1$.

In this paper, we study the case where $F$ has a singularity at the origin. The model example is $F(s)=-|s|^{p-1}$, for $0<p<1$. Our motivation for this study is the following. Mathieu and Saly [11] proposed the fractional power nonlinearity as a model of strong interaction of particles. On the basis of numerical investigations, they observed that the singularity of the self-interaction produces a strong self-confining mechanism, in the sense that the solutions have compact support. In addition, they recovered the MIT bag model (see Chodos, Jaffe, Johnson, Thorn and Weisskopf [5] and Johnson [9]) as a limiting case. Later, Mathieu [10] clarified the relation with the bag model in the framework of a semiclassical approximation.

Our paper is concerned with the mathematical problems related to the existence of confined standing wave solutions to a class of nonlinear classical Dirac fields, including the fractional power model. In this context, we provide a rigorous mathematical solution to the confinement problem raised by Mathieu and Saly. In particular, we prove the existence of solutions having compact support, for the fractional power model. Furthermore, we extend the previous results to more general nonlinearities by obtaining a necessary and sufficient condition for the existence of confined solutions. A simple criterion is obtained, related to an integral condition on the nonlinear term. Essentially, the nonlinearity needs to be singular enough at the origin, in order to produce confinement. We also give a lower bound on the radius of the confined solutions. On the other hand, the mathematical analysis of the confined solitary waves is a basic preliminary step to study their stability, as it happens in the Klein-Gordon field. 
Following Wakano [18] and Soler [10,11] (see also [17]), we seek solutions that are separable in spherical coordinates, of the form

$$
\varphi(x)=\left(\begin{array}{c}
v(r)\left(\begin{array}{c}
0 \\
1
\end{array}\right) \\
i u(r)\left(\begin{array}{c}
\cos \theta \\
\sin \theta e^{i \phi}
\end{array}\right)
\end{array}\right)
$$

Here $r=|x|$ and $(\theta, \phi)$ are the angular parameters. Equation (1.2) then becomes a nonautonomous planar dynamical system in the $r$ variable, which is (compare $[18,15,17])$

$$
\begin{aligned}
u^{\prime}+\frac{2 u}{r} & =v\left(F\left(v^{2}-u^{2}\right)-(M-\omega)\right) ; \\
v^{\prime} & =u\left(F\left(v^{2}-u^{2}\right)-(M+\omega)\right) .
\end{aligned}
$$

In order to eliminate singularities at the origin, due to the term $\frac{2 u}{r}$ in (1.4) we impose the condition

$$
u(0)=0,
$$

and since we are interested in finite energy solutions of (1.2), we seek solutions of (1.4)-(1.5) that verify

$$
\lim _{r \rightarrow \infty}|u(r)|+|v(r)|=0 .
$$

We assume from now on that $F:(0, \infty) \rightarrow(-\infty, 0)$ is $C^{1}$ and satisfies the following assumption:

$$
\left\{\begin{array}{l}
F \text { is integrable near the origin; } \\
F \text { is negative and nondecreasing; } \\
\text { there exists } a \in(0, \infty) \text { such that } F\left(a^{2}\right)=M-\omega \text { and } F^{\prime}\left(a^{2}\right)>0 .
\end{array}\right.
$$

Before proceeding further, let us indicate that we will construct solutions with compact support, if $F$ is sufficiently singular at the origin. Therefore, we must define precisely the type of solutions that we will consider.

Definition 1.1. $(u, v)$ is an admissible positive solution of system (1.4)-(1.7) if there exists a real number $0<R \leqq \infty$ such that the following holds;

(i) $u, v \in C^{1}([0, R))$;

(ii) $0<u(r)<v(r)$, for all $r \in(0, R)$;

(iii) $u(0)=0$;

(iv) $v(r) \underset{r \uparrow R}{\longrightarrow} 0$;

(v) $(u, v)$ solves system $(1.4)-(1.5)$ on $(0, R)$.

Clearly, the number $R$ is unique, and is called the radius of the solution $(u, v)$.

Definition 1.1 deserves some comments. Assumption (iii) eliminates singularities at the origin, and assumption (iv) means that $(u, v)$ vanishes as $r \uparrow R$. Finally, in order that system (1.4)-(1.5) makes sense, it is necessary that $(u, v)$ verifies (i) and that $v^{2}>u^{2}$ on $[0, R)$. That last property is equivalent to assumption (ii) (compare 
Remark 2.8) provided $v(0)>0$, which we always may assume, due to the symmetries of the problem.

Our main result is the following.

Theorem 1.2. Let $0<M<\omega$, and assume that $F$ satisfies assumption (1.8). Then, system (1.4)-(1.7) has an admissible positive solution.

Admissible solutions may have finite or infinite radius, depending on the nonlinear interaction $F$. Let us define the function $G \in C([0, \infty)) \cap C^{1}(0, \infty)$ by

$$
G(x)=-\int_{0}^{x} F(s) d s, \quad \text { for } \quad x \geqq 0,
$$

and the (possibly infinite) number $v$ by

$$
v=\int_{0}^{1} \frac{d s}{G(s)}
$$

Then, we have the following result.

Theorem 1.3. Consider $0<M<\omega$, and assume that $F$ satisfies assumption (1.8). Let $(u, v)$ be an admissible positive solution of system (1.4)-(1.7), and let $R$ be its radius. Then,

(i) $R>\frac{1}{\omega}$;

(ii) $R$ is finite if, and only if $v$ is finite.

Property (ii) of Theorem 1.3 gives a necessary and sufficient condition on $F$ that ensures that all admissible positive solutions of (1.4)-(1.7) have compact support. Property (i) gives a lower bound on the radius of the solutions, depending only on the frequency $\omega$.

So far, we have stated the existence of a solution of system (1.4)-(1.7), and a criterion to determine whether or not that solution has compact support. We will now specify in what sense we have solved the original equation (1.2). We begin with the regular case when the radius of solutions is infinite.

Theorem 1.4. Consider $0<M<\omega$, and assume that $F$ satisfies assumption (1.8). Assume further that $v$ defined by $(1.10)$ is infinite. Let $(u, v)$ be an admissible positive solution of system (1.4)-(1.7) (the radius of $(u, v)$ is infinite, by Theorem 1.3), and let $\varphi$ be defined by (1.3). Then, $\varphi \in C^{1}\left(\mathbf{R}^{3}, \mathbf{C}^{4}\right), \varphi$ solves Eq. (1.2), and $\varphi$ has an exponential fall-off as $|x| \rightarrow \infty$. Furthermore, $|\nabla \varphi|$ is integrable on $\mathbf{R}^{3}$.

Remark 1.5. Note that Eq. (1.2) makes sense. Indeed, $v^{2}-u^{2}>0$ on [0, $\infty$ ), which implies that $\bar{\varphi} \varphi>0$ on $\mathbf{R}^{3}$; and so, $F(\bar{\varphi} \varphi)$ is well defined.

In the case when the admissible solutions have finite radius, we must extend them for larger values for $r$. We proceed as follows. Given an admissible positive solution $(u, v)$ of $(1.4)-(1.7)$ with the finite radius $R$, consider the functions $\hat{u}, \hat{v} \in C([0, \infty))$ defined by

$$
\hat{u}(r)=\left\{\begin{array}{ll}
u(r), & \text { if } \quad 0 \leqq r<R ; \\
0, & \text { if } \quad r>R ;
\end{array} \quad \hat{v}(r)=\left\{\begin{array}{lll}
v(r), & \text { if } & 0 \leqq r<R \\
0, & \text { if } & r>R .
\end{array}\right.\right.
$$


Let $\varphi \in C\left(\mathbf{R}^{3}, \mathbf{C}^{4}\right)$ be defined by

$$
\varphi(x)=\left(\begin{array}{c}
\hat{v}(r)\left(\begin{array}{l}
0 \\
1
\end{array}\right) \\
i \hat{u}(r)\left(\begin{array}{c}
\cos \theta \\
\sin \theta e^{i \phi}
\end{array}\right)
\end{array}\right)
$$

Finally, we define the function $f$ by

$$
f(\varphi)=\left\{\begin{array}{llc}
F(\bar{\varphi} \varphi) \varphi, & \text { if } & \bar{\varphi} \varphi>0 \\
0, & \text { if } & \bar{\varphi} \varphi=0
\end{array}\right.
$$

Then, we have the following result.

Theorem 1.6. Consider $0<M<\omega$, and assume that $F$ satisfies assumption (1.8). Assume further that $v$ defined by $(1.10)$ is finite. Let $(u, v)$ be an admissible positive solution of system (1.4)-(1.7) (the radius of $(u, v)$ is finite, by Theorem 1.3), and let $\varphi$ be defined by (1.11)-(1.12). Then, $\varphi \in W^{1,1}\left(\mathbf{R}^{3}, \mathbf{C}^{4}\right) \cap C\left(\mathbf{R}^{3}, \mathbf{C}^{4}\right), f(\varphi) \in L^{1}\left(\mathbf{R}^{3}, \mathbf{C}^{4}\right)$, and $\varphi$ solves equation

$$
i \sum_{k=1}^{3} \gamma^{k} \partial_{k} \varphi-M \varphi+\omega \gamma^{0} \varphi+f(\varphi)=0
$$

in $L^{1}\left(\mathbf{R}^{3}, \mathbf{C}^{4}\right)$. Furthermore, $\varphi \in C^{1}\left(\mathbf{R}^{3}, \mathbf{C}^{4}\right)$ if and only if $\frac{d}{d s}(G(s))^{3 / 2} \rightarrow 0$, as $s \downarrow 0$.

Here, $L^{1}\left(\mathbf{R}^{3}, \mathbf{C}^{4}\right)$ is the space of integrable functions $\mathbf{R}^{3} \rightarrow \mathbf{C}^{4}$, and $W^{1,1}\left(\mathbf{R}^{3}, \mathbf{C}^{4}\right)$ is the Sobolev space of functions of $L^{1}$ whose first derivatives (in the sense of distributions) belong to $L^{1}$.

Note that Eq. (1.2) makes sense (see Remark 1.5). Furthermore, note that $\varphi$ is $C^{1}$ for $|x|<R$ and $|x|>R$. Therefore, the possible discontinuities of $\nabla \varphi$ are located on the sphere $\{|x|=R\}$ (see Lemma 4.4 for more information on the nature of the singularity).

We will now apply our results to some model nonlinearities.

Example 1. Take $F(s)=-\frac{1}{1+s^{2}}$, and consider $0<M<\omega<M+1$. Then, there exists a solution $\varphi \in C^{1}\left(\mathbf{R}^{3}, \mathbf{C}^{4}\right)$ of Eq. (1.2), such that $\varphi$ has an exponential fall-off as $|x| \rightarrow \infty$. It follows from the equation that $\nabla \varphi$ also has an exponential fall-off as $|x| \rightarrow \infty$. In addition, $\bar{\varphi} \varphi>0$ on $\mathbf{R}^{3}$.

Example 2. Let $F:(0, \infty) \rightarrow(-\infty, 0)$ be an increasing function such that $F(s) \rightarrow 0$, as $s \rightarrow \infty$, and such that $F(s)=\log (s)$, for $s$ small. Consider $0<M<\omega$. Then, there exists a solution $\varphi \in C^{1}\left(\mathbf{R}^{3}, \mathbf{C}^{4}\right)$ of Eq. (1.2), such that $\varphi$ has an exponential fall-off as $|x| \rightarrow \infty$ and $\nabla \varphi \in L^{1}\left(\mathbf{R}^{3}, \mathbf{C}^{4}\right)$. In addition, $\bar{\varphi} \varphi>0$ on $\mathbf{R}^{3}$.

Example 3. Let $F(s)=-s^{-\alpha}$, for some $0<\alpha<1 / 3$, and consider $0<M<\omega$. Then, there exists a solution $\varphi \in C^{1}\left(\mathbf{R}^{3}, \mathbf{C}^{4}\right)$ of Eq. (1.2), with compact support.

Example 4. Let $F(s)=-s^{-\alpha}$, for some $1 / 3<\alpha<1$, and consider $0<M<\omega$. Then, there exists a solution $\varphi \in W^{1,1}\left(\mathbf{R}^{3}, \mathbf{C}^{4}\right)$ of Eq. (1.2), with compact support. In addition, $\varphi$ is continuously differentiable, except on some sphere $\{|x|=R\}$, where 
$\nabla \varphi$ is infinite (see Remark 4.5). That property was observed by Mathieu and Saly [11].

Note that these results are quite similar to those concerning the Klein-Gordon field (see Stubbe [16]). However, the solutions of the Klein-Gordon equation have less singularities than the solutions of Dirac's equation. For example, when $F(s)=-s^{-\alpha}$, for $1 / 3<\alpha<1$, the solutions of Dirac's equation have a discontinuous derivate, while the solutions of the Klein-Gordon equation are of class $C^{2}$ (see Stubbe [16]).

The paper is organized as follows. In Sect. 2, we define the notation and we establish preliminary results concerning the dynamical system (1.4)-(1.5). In particular, we study the behaviour of the solutions near the diagonal $\{u=v\}$ where the system becomes singular. Section 3 is devoted to the proof of Theorem 1.2 and Sect. 4 to the proof of Theorems 1.3, 1.4 and 1.6.

The authors wish to express their thanks to J. Stubbe for valuable discussions.

\section{Notations and Preliminary Results}

In all that follows, we consider $0<M<\omega$, and we assume that $F$ verifies (1.8). Let

$$
F_{\infty}=\lim _{s \rightarrow \infty} F(s) \leqq 0 .
$$

For convenience, we set

$$
m=M-F_{\infty} \geqq M,
$$

and

$$
\phi(s)=-\left(F(s)-F_{\infty}\right), \text { for } s>0,
$$

so that $\phi(s) \geqq 0$ for $s>0$. We also set

$$
\phi(s)=\int_{0}^{s} \phi(\sigma) d \sigma, \quad \text { for } \quad s \geqq 0 .
$$

Observe that (1.4)-(1.15) is equivalent to the following system:

$$
\begin{aligned}
u^{\prime}+\frac{2 u}{r} & =v\left(-\phi\left(v^{2}-u^{2}\right)-(m-\omega)\right), \\
v^{\prime} & =u\left(-\phi\left(v^{2}-u^{2}\right)-(m+\omega)\right),
\end{aligned}
$$

Remark 2.1. Note that by assumption (1.8), we have the following properties.

(i) $0<m<\omega$;

(ii) $\phi\left(a^{2}\right)=\omega-m$;

(iii) $\phi(s) \geqq \phi\left(s_{0}\right)>\omega-m$, for $0<s \leqq s_{0}<a^{2}$;

(iv) $\phi(s) \underset{s \rightarrow+\infty}{\longrightarrow} 0$.

We define the continuous function $H$ on the set $\left\{v^{2} \geqq u^{2}\right\}$ by

$$
H(u, v)=-\frac{1}{2} \Phi\left(v^{2}-u^{2}\right)+\frac{1}{2}(\omega-m)\left(v^{2}-u^{2}\right)+\omega u^{2},
$$


and for $\tau>0$, we define the continuous function $\tilde{H}_{\tau}$ on the set $\left\{v^{2} \geqq u^{2}\right\}$ by

$$
\begin{aligned}
\tilde{H}_{\tau}(u, v) & =-\frac{1}{2} \Phi\left(v^{2}-u^{2}\right)-\frac{1}{2}\left(m+\frac{1}{\tau}\right)\left(v^{2}-u^{2}\right)+\frac{1}{2}\left(\omega-\frac{1}{\tau}\right)\left(v^{2}+u^{2}\right) \\
& =H(u, v)-\frac{1}{\tau} v^{2} .
\end{aligned}
$$

For convenience, we set

$$
\tilde{H}_{\infty}=H .
$$

Note that $H$ and $\tilde{H}_{\tau}$ are $C^{2}$ on the set $\left\{v^{2}>u^{2}\right\}$. Given $x \neq 0$, we write the system

$$
\left\{\begin{array}{l}
(2.1),(2.2) \\
u(0)=0, \quad v(0)=x
\end{array}\right.
$$

in the integral form

$$
\left\{\begin{array}{l}
u(r)=\frac{1}{r^{2}} \int_{0}^{r} s^{2} v(s)\left(-\phi\left(v^{2}(s)-u^{2}(s)\right)-(m-\omega)\right) d s \\
v(r)=x+\int_{0}^{r} u(s)\left(-\phi\left(v^{2}(s)-u^{2}(s)\right)-(m+\omega)\right) d s
\end{array}\right.
$$

Since the integrand is a locally Lipschitz continuous function of $(u, v)$ on the open set $\left\{v^{2}>u^{2}\right\}$ where $\phi\left(v^{2}-u^{2}\right)$ is defined and $C^{1}$, existence of a maximal solution follows from the classical contraction mapping argument. It follows that there exists a unique maximal solution $\left(u_{x}, v_{x}\right) \in C^{1}\left(\left[0, R_{x}\right), \mathbf{R}^{2}\right)$ of $(2.3)$, such that $v_{x}^{2}-u_{x}^{2}>0$ on $\left[0, R_{x}\right)$. Moreover, for $R<R_{x},\left(u_{x}, v_{x}\right)$ depends continuously on $x \neq 0$ in the $C^{1}\left([0, R], \mathbf{R}^{2}\right)$ topology. In addition, if $R_{x}<\infty$ then $u_{x}^{2}-v_{x}^{2} \rightarrow 0$ or $\left|u_{x}\right|+\left|v_{x}\right| \rightarrow \infty$, as $r \rightarrow R_{x}$. We define the orbit $\Gamma_{x}$ of $(0, x)$ by

$$
\Gamma_{x}=\left\{\left(u_{x}(r), v_{x}(r)\right), r \in\left[0, R_{x}\right)\right\} \text {. }
$$

We will need the following straightforward properties of $\Gamma_{x}$.

Lemma 2.2. Let $x \neq 0$ and $r \in\left[0, R_{x}\right)$. We have

(i) $\frac{d}{d r} H\left(u_{x}(r), v_{x}(r)\right)=\frac{2}{r} u_{x}^{2}\left(-\phi\left(v_{x}^{2}-u_{x}^{2}\right)-(m+\omega)\right) \leqq 0$;

(ii) $\frac{d}{d r} \tilde{H}_{\tau}\left(u_{x}(r), v_{x}(r)\right)=2 u_{x}\left(-\phi\left(v_{x}^{2}-u_{x}^{2}\right)-(m+\omega)\right)\left(\frac{u_{x}}{r}-\frac{v_{x}}{\tau}\right)$; in particular, if $u \geqq 0$, then $\frac{d}{d r} \tilde{H}_{\tau}\left(u_{x},(r), v_{x}(r)\right) \leqq 0$, if and only if $\frac{u_{x}}{r}-\frac{v_{x}}{\tau} \geqq 0$.

In view of Lemma 2.2, several important properties of $\Gamma_{x}$ follows from the structure of the sets $\{H(u, v)=C\}$ and $\left\{\tilde{H}_{\tau}(u, v)=C\right\}$. We will study those sets in the following lemmas:

Lemma 2.3. Let $\frac{2}{\omega-m}<\tau \leqq \infty$. Then,

(i) there exists two constants $C_{\tau}>0$ and $A_{\tau}>0$ such that

$$
\tilde{H}_{\tau}(u, v) \geqq C_{\tau}\left(u^{2}+v^{2}\right)-A_{\tau} \quad \text { for } \quad v^{2} \geqq u^{2} ;
$$


in particular, the set $\left\{\tilde{H}_{\tau}(u, v) \leqq K\right\}$ is bounded, for every $K$;

(ii) $\tilde{H}_{\tau}(0, v)<0$, for all $v \in(0, a]$;

(iii) there exists $\eta_{\tau}>0$ such that if $\tilde{H}_{\tau}(u, v) \geqq 1$ for some $0 \leqq u \leqq v$, then $v \geqq \eta_{\tau}$.

Proof. (i) It follows from Remark 2.1 that

$$
\limsup _{s \rightarrow \infty} \frac{1}{s} \Phi(s)=0 .
$$

Therefore, for every $\varepsilon>0$, there exists a constant $K_{\varepsilon}$ such that

It follows that

$$
\Phi(s) \leqq \varepsilon s+K_{\varepsilon}, \quad \text { for every } s \geqq 0 .
$$

$$
\begin{aligned}
\tilde{H}_{\tau}(u, v) & \geqq-\frac{1}{2}\left(m+\varepsilon+\frac{1}{\tau}\right)\left(v^{2}-u^{2}\right)+\frac{1}{2}\left(\omega-\frac{1}{\tau}\right)\left(v^{2}+u^{2}\right)-\frac{K_{\varepsilon}}{2} \\
& \geqq \frac{1}{2}\left(\omega-m-\frac{2}{\tau}-\varepsilon\right)\left(v^{2}+u^{2}\right)-\frac{K_{\varepsilon}}{2} .
\end{aligned}
$$

(i) follows by choosing $0<\varepsilon<\omega-m-\frac{2}{\tau}$.

(ii) Let $0 \leqq v \leqq a$. By Remark 2.1 , we have $\phi \geqq \omega-m$ on $\left[0, a^{2}\right]$; and so $\Phi\left(v^{2}\right) \geqq(\omega-\bar{m}) v^{2}$. It follows that

$$
\tilde{H}_{\tau}(0, v) \leqq-\frac{1}{2}(\omega-m) v^{2}-\frac{1}{2}\left(m+\frac{1}{\tau}\right) v^{2}+\frac{1}{2}\left(\omega-\frac{1}{\tau}\right) v^{2}=-\frac{v^{2}}{\tau} \leqq 0 .
$$

Note also that for $v>0$, the first inequality is strict. Hence (ii).

(iii) follows from continuity and the fact that $\widetilde{H}_{\tau}(0,0)=0$.

Lemma 2.4. Let $\frac{1}{\omega}<\tau<\frac{2}{\omega-m}$ and let $G_{\tau}=\left\{0 \leqq u \leqq v, \tilde{H}_{\tau}(u, v)=0\right\}$. Then,

(i) for $(u, v) \in G_{\tau}$ such that $u>0$, we have $\tilde{H}_{\tau}(z, v)<0$, for $0 \leqq z<u$;

(ii) for $(u, v) \in G_{\tau}$ such that $u+v>0$, we have $u<v$;

(iii) $G_{\tau}$ is a connected unbounded curve containing the origin;

(iv) $G_{\tau}$ is tangent to $\{u=v\}$ at the origin, if $\phi(0)=\infty$;

(v) for $(u, v) \in G_{\tau}$ such that $u+v \rightarrow \infty$, we have $\frac{u^{2}}{v^{2}} \rightarrow \frac{\frac{2}{\tau}-(\omega-m)}{\omega+m} \in(0,1)$.

Proof. Note that

$$
\begin{aligned}
\tilde{H}_{\tau}(0,0) & =0 \\
\tilde{H}_{\tau}(v, v) & =\left(\omega-\frac{1}{\tau}\right) v^{2}>0, \text { for } v>0 ; \\
\tilde{H}_{\tau}(0, v) & =-\Phi\left(v^{2}\right)-\left(\frac{2}{\tau}+m-\omega\right) v^{2}<0, \text { for } v>0 ; \\
\frac{d}{d\left(u^{2}\right)} \tilde{H}_{\tau}(u, v) & =\frac{1}{2} \phi\left(v^{2}-u^{2}\right)+\frac{1}{2}(m+\omega) .
\end{aligned}
$$


This implies (i), (ii) and (iii).

To prove (iv), observe that for $(u, v) \in G_{\tau}$,

$$
0=-\frac{\Phi\left(v^{2}-u^{2}\right)}{v^{2}-u^{2}}-m-\frac{1}{\tau}+\left(\omega-\frac{1}{\tau}\right) \frac{v^{2}+u^{2}}{v^{2}-u^{2}}
$$

(iv) follows, since $\phi$ is singular at the origin.

To prove (v), observe that for $(u, v) \in G_{\tau}$,

$$
0=-\frac{\Phi\left(v^{2}-u^{2}\right)}{v^{2}}+\frac{2}{\tau}-\omega+m+(\omega+m) \frac{u^{2}}{v^{2}} \text {. }
$$

Note also that

$$
0 \leqq \frac{\Phi\left(v^{2}-u^{2}\right)}{v^{2}} \leqq \frac{\Phi\left(v^{2}\right)}{v^{2}} \underset{v^{2} \rightarrow \infty}{\longrightarrow} 0
$$

Hence (v).

We continue with two simple applications of Lemma 2.2.

Corollary 2.5. For every $x \neq 0$ we have $\sup \left\{\left|u_{x}(r)\right|+\left|v_{x}(r)\right|, r \in\left[0, R_{x}\right)\right\}<\infty$, and if $R_{x}<\infty$, then $u_{x}^{2}-v_{x}^{2} \rightarrow 0$ as $r \rightarrow R_{x}$.

Proof. By property (i) of Lemma 2.2 and the local existence argument, it is sufficient to show that for any given $C$, the set $\left\{(u, v) \in \mathbf{R}^{2}, v^{2}>u^{2}\right.$ and $\left.H(u, v) \leqq C\right\}$ is bounded. This follows from Lemma 2.3 (i), applied with $\tau=\infty$.

Corollary 2.6. Let $x^{2} \geqq a^{2}$. Then $u_{x}^{2}<v_{x}^{2} \leqq x^{2}$ on $\left[0, R_{x}\right)$.

Proof. By property (i) of Lemma 2.2, we have

$$
H\left(u_{x}, v_{x}\right) \leqq H(0, x), \quad \text { on } \quad\left[0, R_{x}\right) .
$$

On the other hand, note that

$$
\frac{d}{d\left(u^{2}\right)} \tilde{H}_{\tau}(u, v)=\frac{1}{2} \phi\left(v^{2}-u^{2}\right)+\frac{1}{2}(m+\omega) \geqq 0 ;
$$

and so,

$$
H\left(0, v_{x}\right) \leqq H\left(u_{x}, v_{x}\right) \leqq H(0, x), \quad \text { on } \quad\left[0, R_{x}\right) .
$$

Since $2 H(0, v)=(\omega-m) v^{2}-\Phi\left(v^{2}\right)$ is a nondecreasing function of $v^{2}$ on $\left[a^{2}, \infty\right)$, it follows that $v_{x}^{2} \leqq x^{2}$ on $\left[0, R_{x}\right)$. Inequality $u_{x}^{2}<v_{x}^{2}$ follows from the definition of $R_{x}$. Proposition 2.7. There exist $\alpha>0$ and $\delta \in\left(0, \frac{m+\omega}{2}\right)$ with the following property.
For every $x \neq 0$, we have

(i) $R_{x}>\frac{1}{\omega-\delta}$;

(ii) $v_{x}^{2}-u_{x}^{2} \geqq \alpha x^{2}$ on $\left[0, \frac{1}{\omega-\delta}\right)$. 
Proof. Let $\delta \in\left(0, \frac{m+\omega}{2}\right)$ be such that

$$
e^{-(4 \omega / \omega-\delta)}\left(1+\frac{\delta}{\omega}\right)>\frac{\delta}{\omega}
$$

let

$$
\alpha=e^{-(4 \omega / \omega-\delta)}\left(1+\frac{\delta}{\omega}\right)-\frac{\delta}{\omega}>0,
$$

and

$$
\rho_{x}=\min \left\{R_{x}, \frac{1}{\omega-\delta}\right\} .
$$

For $0 \leqq r \leqq \rho_{x}$, we have by Corollary 2.5 ,

$$
\begin{aligned}
\frac{d}{d r}\left(v_{x}^{2}-u_{x}^{2}\right) & =2\left(v_{x} v_{x}^{\prime}-u_{x} u_{x}^{\prime}\right)=4\left(\frac{u_{x}^{2}}{r}-\omega u_{x} v_{x}\right) \\
& \geqq 4\left((\omega-\delta) u_{x}^{2}-\omega v_{x}^{2}\right)=4 \omega\left(u_{x}^{2}-v_{x}^{2}\right)-4 \delta u_{x}^{2} \\
& \geqq 4 \omega\left(u_{x}^{2}-v_{x}^{2}\right)-4 \delta x^{2}
\end{aligned}
$$

It follows that

$$
\frac{d}{d r}\left(e^{4 \omega r}\left(v_{x}^{2}-u_{x}^{2}\right)\right)+4 \delta x^{2} e^{4 \omega r} \geqq 0, \quad \text { on } \quad\left[0, \rho_{x}\right)
$$

Integrating the above equation yields

$$
v_{x}^{2}-u_{x}^{2} \geqq \alpha x^{2}, \quad \text { on }\left[0, \rho_{x}\right) .
$$

In particular, $v_{x}^{2}-u_{x}^{2}>0$ on $\left[0, \rho_{x}\right)$. When Corollary 2.5 is applied, this implies that $\rho_{x}<R_{x}$. Hence the result.

Remark 2.8. Consider $x>0$, and assume that $v_{x}(r) \rightarrow 0$, as $r \uparrow R_{x}$. Then, $\left(u_{x}, v_{x}\right)$ is an admissible positive solution, with radius $R_{x}$. Indeed, in view of Definition 1.1, it is sufficient to verify that $u_{x}>0$ on $\left(0, R_{x}\right)$. Note that $H\left(u_{x}(r), v_{x}(r)\right) \rightarrow 0$, as $r \uparrow R_{x}$. It follows Lemma 2.2 that $H\left(u_{x}(r), v_{x}(r)\right)>0$ on $\left[0, R_{x}\right)$. Therefore, if $u_{x}(\rho)=0$ for some $\rho \in\left[0, R_{x}\right)$, we have $v_{x}(\rho)>a$ by Lemma 2.3 , and it follows from Eq. (2.1) that $u_{x}^{\prime}(\rho)>0$; and so, we must have $\rho=0$. Hence the result. Note that we must have $x>a$.

\section{Proof of Theorem 1.2}

Consider $x>a$. It follows from (2.1) that $u_{x}^{\prime}(0)>0$. Therefore we have $u_{x}>0$ and $v_{x}>0$ for $r$ small. Note that by Corollary $2.6, v_{x}$ cannot vanish on $\left[0, R_{x}\right)$; and so $v_{x}>0$ on $\left[0, R_{x}\right)$. Let

$$
r_{x}=\sup \left\{r \in\left(0, R_{x}\right), u_{x}>0 \text { on }(0, r)\right\} .
$$

Clearly, we have $0<u_{x}<v_{x}$ on $\left(0, r_{x}\right)$. Furthermore, either $r_{x}=R_{x}$, or else $r_{x}<R_{x}$ 
and then $u_{x}\left(r_{x}\right)=0$ and $v_{x}\left(r_{x}\right)>0$. Note that in the latter case, we must have $u_{x}^{\prime}\left(r_{x}\right) \leqq 0$, which implies in view of $(2.2)$ that

$$
0<v_{x}\left(r_{x}\right)<a \text {. }
$$

We define the set $I_{0}$ as follows:

$$
\begin{aligned}
I_{0} & =\left\{x \in(a, \infty), r_{x}<R_{x}\right\} \\
& =\left\{x \in(a, \infty), 0<u_{x}<v_{x} \text { on }\left(0, r_{x}\right), v_{x}\left(r_{x}\right) \in(0, a), u_{x}\left(r_{x}\right)=0\right\} .
\end{aligned}
$$

The strategy for proving Theorem 1.2 is the following. We will first show that $I_{0}$ is a nonempty, bounded, open subset of $R$. The open character of $I_{0}$ is a simple consequence of the continuous dependence of the solutions on the initial data, the nonemptyness follows essentially from a result of Cazenave and Vázquez [4], but the boundedness requires the rather delicate construction of a trapping region. The next step consists in showing that if we set $y=\sup I_{0}$, then $\left|u_{y}\right|+\left|v_{y}\right| \rightarrow 0$ as $r \rightarrow R_{y}$. Extending $\left(u_{x}, v_{x}\right)$ by $(0,0)$ in $\left(R_{y}, \infty\right)$ yields the desired solution. This result relies also on the construction of a trapping region that prevents the solutions with initial data in $I_{0}$ to come too close to the set $\{u=v\}$ except at the origin. The further properties of the solution follow from standard estimates.

Lemma 3.1. $I_{0}$ is nonempty. More precisely, every $x>$ a such that $H(0, x)<0$ belongs to $I_{0}$.

Proof. Consider $x>a$ such that $H(0, x)=-h<0$ (such as $x$ exists by Lemma 2.3(ii)). By Lemma 2.2 , we have $H\left(u_{x}, v_{x}\right) \leqq-h$ on $\left[0, R_{x}\right)$. Since $H(v, v)=\omega v^{2} \geqq 0$, it follows that $\Gamma_{x}$ is bounded away from the set $\left\{u^{2}=v^{2}\right\}$ and the result is a consequence of the following Proposition.

Proposition 3.2. Let $x>a$. Assume that there exists $\varepsilon>0$ such that $v_{x}^{2}-u_{x}^{2} \geqq \varepsilon$ on $\left[0, R_{x}\right)$. Then $x \in I_{0}$.

Proof. By Corollary 2.5, we have $R_{x}=\infty$. Thus we only have to prove that $r_{x}<\infty$. We argue by contradiction and we suppose on the contrary that $r_{x}=\infty$. It follows that

$$
0<u_{x}<v_{x} \leqq x \text { on }[0, \infty) \text {, }
$$

where the last inequality follows from Corollary 2.6. Note also that by (2.2) $v_{x}$ is decreasing. Therefore, there exists $v$ such that

$$
v_{x} \downarrow v>0 \text {, as } r \rightarrow \infty \text {, }
$$

and $v>0$ since $H\left(u_{x}, v_{x}\right) \leqq-h$. Note that by (2.1)-(2.2),

$$
\frac{d}{d r}\left(v_{x}^{2}-u_{x}^{2}\right)=2 u_{x}\left(\frac{u_{x}}{r}-\omega v_{x}\right) \text {. }
$$

Since $\omega v_{x} \rightarrow \omega v>0$ and $\frac{u_{x}}{r} \rightarrow 0$, as $r \rightarrow \infty$, it follows that $v_{x}^{2}-u_{x}^{2}$ is decreasing for $r$ large; and since $v_{x}^{2}-u_{x}^{2} \geqq \varepsilon>0$, there exists $l>0$ such that $v_{x}^{2}-u_{x}^{2} \downarrow l$, as $r \rightarrow \infty$. It follows that $u_{x}$ also has a limit $u \geqq 0$, as $r \rightarrow \infty$. By (2.2), we have

$$
v_{x}^{\prime}(r) \underset{r \rightarrow \infty}{\longrightarrow} u(-\phi(l)-m-\omega) \leqq-(m+\omega) u .
$$


Since $v_{x}$ has a limit, we must have $u=0$. Now, by (2.1), we have

$$
u_{x}^{\prime}(r) \underset{r \rightarrow \infty}{\longrightarrow} v\left(-\phi\left(v^{2}\right)-m+\omega\right)
$$

and so, we must have $v\left(-\phi\left(v^{2}\right)-m+\omega\right)=0$, which means $v=a$. Thus,

$$
\left(u_{x}, v_{x}\right) \rightarrow(0, a), \text { as } r \rightarrow \infty \text {. }
$$

Let $(U, V)=\left(u_{x}, v_{x}-a\right)$. By assumption $(U, V) \rightarrow(0,0)$ as $r \rightarrow \infty$ and $U, V>0$. The equations for $U$ and $V$ are

$$
\left\{\begin{array}{l}
U^{\prime}=-\frac{2 U}{r}+(a+V)\left(-\phi\left(a^{2}+V^{2}+2 a V-U^{2}\right)-m+\omega\right), \\
V^{\prime}=U\left(-\phi\left(a^{2}+V^{2}+2 a V-U^{2}\right)-m-\omega\right) .
\end{array}\right.
$$

We have $V^{2}+2 a V-U^{2} \rightarrow 0$, and $\left|V^{2}+2 a V-U^{2}\right| \leqq C(U+V)$. Therefore if we set

$$
-d=\phi^{\prime}\left(a^{2}\right)<0 \text {, }
$$

we have

$$
\phi\left(a^{2}+V^{2}+2 a V-U^{2}\right)=\omega-m-2 a d V+o(U+V) .
$$

Equation (3.2) becomes then

$$
\left\{\begin{array}{l}
U^{\prime}=-\frac{2 U}{r}+2 a^{2} d V+o(U+V) \\
V^{\prime}=-2 \omega U+o(U+V)
\end{array}\right.
$$

It follows that

$$
(V-U)^{\prime}=\omega(V-U)-\left(\omega-\frac{2}{r}\right) U-\left(\omega+2 a^{2} d\right) V+o(U+V), \quad \text { for } \quad r \text { large. }
$$

Therefore $e^{-\omega r}(V-U)$ is nonincreasing for $r$ large. Since $e^{-\omega r}(V-U) \rightarrow 0$, as $r \rightarrow \infty$, it follows that $V-U \geqq 0$ for $r$ large. Thus we can replace $o(U+V)$ by $o(V)$ and we get from the first equation in (3.3),

$$
U^{\prime}=a^{2} d V-\frac{2}{r} U+o(V) \geqq \frac{a^{2} d}{2} V>0, \text { for } \quad r \text { large. }
$$

Thus $U$ is increasing for $r$ large, which is a contradiction since $U>0$ and $U \rightarrow 0$. Hence the result.

Lemma 3.3. $I_{0}$ is an open subset of $\mathbf{R}$.

Proof. Let $x_{0} \in I_{0}$. We have $0<u_{x_{0}}<v_{x_{0}}<x_{0}$ on $\left(0, r_{x_{0}}\right)$ and $0<v_{x_{0}}\left(r_{x_{0}}\right)<a$ on $\left[0, r_{x_{0}}\right]$. It follows in particular that $u_{x_{0}}^{\prime}\left(r_{x_{0}}\right)<0$; and so $u_{x_{0}}$ becomes negative after $r_{x_{0}}$. Therefore, it follows easily from the continuous dependence of $\left(u_{x}, v_{x}\right)$ on $\mathrm{x}$ that for $x$ close enough to $x_{0}$, we have $x \in I_{0}$. Hence the result.

Proposition 3.4. $I_{0}$ is bounded.

Before proceeding to the proof of Proposition 3.4, we will establish the following preliminary lemma. 
Lemma 3.5. Let $\alpha$ and $\delta$ be defined by Proposition 2.7 and let $x \in I_{0}$. Then the following holds.

(i) If $\alpha x^{2}>a^{2}$, then $r_{x}>\frac{1}{\omega-\delta}$;

(ii) if $r_{x}>\frac{1}{\omega-\delta}$, then $\tilde{H}_{1 /(\omega-\delta)}\left(u_{x}(r), v_{x}(r)\right) \leqq 0$, for every $r \in\left(\frac{1}{\omega-\delta}, r_{x}\right)$.

Proof. (i) By Proposition 2.7, we have

$$
v_{x}^{2} \geqq v_{x}^{2}-u_{x}^{2} \geqq \alpha x^{2}>a^{2}, \quad \text { for } \quad 0 \leqq r \leqq \frac{1}{\omega-\delta}<R_{x} .
$$

In particular, we have

$$
v_{x}>a, \text { for } 0 \leqq r \leqq \frac{1}{\omega-\delta}<R_{x} .
$$

(i) follows, by (3.1).

(ii) Consider $r \in\left(\frac{1}{\omega-\delta}, r_{x}\right)$. We have

$$
\frac{u_{x}(r)}{r}-(\omega-\delta) v_{x}(r) \leqq(\omega-\delta)\left(u_{x}(r)-v_{x}(r)\right) \leqq 0,
$$

since $x \in I_{0}$. By Lemma 2.2 (ii), this implies that $\tilde{H}_{1 /(\omega-\delta)}\left(u_{x}, v_{x}\right)$ is nondecreasing on $\left(\frac{1}{\omega-\delta}, r_{x}\right)$. Note also that, by Lemma 2.4 (i) and (iii), we have $\tilde{H}_{1 /(\omega-\delta)}(0, v)<0$, for every $v>0$. In particular, $\tilde{H}_{1 /(\omega-\delta)}\left(u_{x}\left(r_{x}\right), v_{x}\left(r_{x}\right)\right)<0$. Hence (ii).

Proof of Proposition 3.4. Let $\alpha$ and $\delta$ be defined by Proposition 2.7 and consider $\tau>\frac{2}{\omega-m}$. Let $x \in I_{0}$. It follows from (3.1) and Lemma 2.3 (ii) that $\tilde{H}_{\tau}\left(u_{x}\left(r_{x}\right), v_{x}\left(r_{x}\right)\right) \leqq 0$. Thus we can define the number $\rho_{x}<r_{x}$ by

$$
\rho_{x}=\inf \left\{r \in\left(0, r_{x}\right), \tilde{H}_{\tau}\left(u_{x}, v_{x}\right) \leqq 1 \text { on }\left[r, r_{x}\right]\right\} .
$$

Note in particular that $\tilde{H}_{\tau}\left(u_{x}\left(\rho_{x}\right), v_{x}\left(\rho_{x}\right)\right) \leqq 1$, and that $\tilde{H}_{\tau}\left(u_{x}\left(\rho_{x}\right), v_{x}\left(\rho_{x}\right)\right)=1$ if $\rho_{x}>0$. Assume first that $\rho_{x} \leqq \frac{1}{\omega-\delta}$. Then, we have by Proposition 2.7 and Lemma 2.3

$$
\alpha x^{2} \leqq v_{x}^{2}\left(\rho_{x}\right)-u_{x}^{2}\left(\rho_{x}\right) \leqq v_{x}^{2}\left(\rho_{x}\right)+u_{x}^{2}\left(\rho_{x}\right) \leqq \frac{A_{\tau}+1}{C_{\tau}},
$$

which implies the desired bound on $x$. Therefore, we can assume that $\rho_{x}>\frac{1}{\omega-\delta}$. Then $\tilde{H}_{\tau}\left(u_{x}\left(\rho_{x}\right), v_{x}\left(\rho_{x}\right)\right)=1$; and so there exists $\sigma \in\left(\rho_{x}, r_{x}\right)$ such that

$$
\frac{d}{d r} \tilde{H}_{\tau}\left(u_{x}, v_{x}\right)(\sigma)<0
$$

By Lemma 2.2 (ii), this implies that $\frac{u_{x}(\sigma)}{\sigma} \geqq \frac{v_{x}(\sigma)}{\tau}$. Since $\sigma<r_{x}$, we have $0<u_{x}(\sigma)<v_{x}(\sigma)$; 
and so we must have $\sigma<\tau$, which implies

$$
\rho_{x}<\tau
$$

Let $\eta_{\tau}$ be defined in Lemma 2.3 (iii) and consider the set

$$
\Lambda=\left\{(u, v) ; u>0, v \geqq \eta_{\tau}, \tilde{H}_{1 /(\omega-\delta)}(u, v) \leqq 0\right\} .
$$

It follows from Lemma 2.3 that $\Lambda$ is bounded away from the set $\{u=v\}$. Therefore, there exists $K_{\tau}$ such that

$$
\phi(u, v) \leqq K_{\tau}, \quad \text { for every }(u, v) \in \Lambda .
$$

Note that by definition of $\rho_{x}$, Lemma 2.3 (iii) and Lemma 3.5 (ii), we have $\left(u_{x}(r), v_{x}(r)\right) \in \Lambda$, for every $r \in\left(\frac{1}{\omega-\delta}, \rho_{x}\right)$. Therefore, applying Lemma 2.2 (ii), (3.5) and Lemma 2.3 (i), we obtain

$$
\begin{aligned}
\frac{d}{d r} \tilde{H}_{\tau}\left(u_{x}(r), v_{x}(r)\right) & \geqq-2\left(\phi\left(v_{x}^{2}-u_{x}^{2}\right)+m+\omega\right) \frac{u_{x}^{2}}{r} \\
& \geqq-\left(\phi\left(v_{x}^{2}-u_{x}^{2}\right)+m+\omega\right)(\omega-\delta) u_{x}^{2} \\
& \geqq-2\left(K_{\tau}+m+\omega\right)(\omega-\delta)\left(u_{x}^{2}+v_{x}^{2}\right) \\
& \geqq-\frac{2}{C_{\tau}}\left(K_{\tau}+m+\omega\right)(\omega-\delta)\left(A_{\tau}+\tilde{H}_{\tau}\left(u_{x}, v_{x}\right)\right) . \\
& =-\beta_{\tau} \tilde{H}_{\tau}\left(u_{x}, v_{x}\right)-\gamma_{\tau},
\end{aligned}
$$

for some constants $\beta_{\tau}$ and $\gamma_{\tau}$ (independent of $x$ ). It follows that

$$
\frac{d}{d r}\left(e^{\beta_{\tau} r} \tilde{H}_{\tau}\left(u_{x}(r), v_{x}(r)\right)\right)+\gamma_{\tau} e^{\beta_{\tau} r} \geqq 0, \quad \text { on }\left(\frac{1}{\omega-\delta}, \rho_{x}\right) .
$$

Integrating the above inequality and applying (3.4), we obtain

$$
\begin{aligned}
\tilde{H}_{\tau}\left(u_{x}\left(\frac{1}{\omega-\delta}\right), v_{x}\left(\frac{1}{\omega-\delta}\right)\right) & \leqq e^{-\beta_{\tau} /(\omega-\delta)} e^{\beta_{\tau} \rho_{x}}\left(\tilde{H}_{\tau}\left(u_{x}\left(\rho_{x}\right), v_{x}\left(\rho_{x}\right)\right)+\frac{\gamma_{\tau}}{\beta_{\tau}}\right) \\
& \leqq e^{-\beta_{\tau} /(\omega-\delta)} e^{\beta_{\tau} \tau}\left(1+\frac{\gamma_{\tau}}{\beta_{\tau}}\right) \leqq L_{\tau}
\end{aligned}
$$

where $L_{\tau}$ does not depend on $x$. It follows from Proposition 2.7 and Lemma 2.3 (i) that

$$
\alpha x^{2} \leqq v_{x}^{2}\left(\frac{1}{\omega-\delta}\right)-u_{x}^{2}\left(\frac{1}{\omega-\delta}\right) \leqq v_{x}^{2}\left(\frac{1}{\omega-\delta}\right)+u_{x}^{2}\left(\frac{1}{\omega-\delta}\right) \leqq \frac{L_{\tau}+A_{\tau}}{C_{\tau}}
$$

Hence the result.

Lemma 3.6. Let $\alpha$ and $\delta$ be defined by Proposition 2.7, let $y=\sup I_{0}$ and let

$$
\Delta=\left\{(u, v) ; 0 \leqq u \leqq v \leqq y, \min \left\{\alpha a^{2}-\left(v^{2}-u^{2}\right), \tilde{H}_{1 /(\omega-\delta)}(u, v)\right\} \leqq 0\right\} .
$$

Then, for every $x \in I_{0}$, we have $\left(u_{x}(r), v_{x}(r)\right) \in \Delta$ for every $r \in\left[0, r_{x}\right]$.

Proof. Let $x \in I_{0}$. We have $0 \leqq u_{x} \leqq v_{x} \leqq x \leqq y$ on $\left[0, r_{x}\right]$. Furthermore, by Proposi- 
tion 2.7, we have $v_{x}^{2}-u_{x}^{2} \geqq \alpha x^{2} \geqq \alpha a^{2}$ on $\left[0, \min \left\{\frac{1}{\omega-\delta}, r_{x}\right\}\right]$. Finally, if $r_{x}>\frac{1}{\omega-\delta}$, we have $\tilde{H}_{1 /(\omega-\delta)}\left(u_{x}, v_{x}\right) \leqq 0$ on $\left[\frac{1}{\omega-\delta}, r_{x}\right]$, by Lemma 3.5 (ii). Hence the result.

Proof of Theorem 1.2. Let $y=\sup I_{0} . y$ is finite by Proposition 3.4. We claim that

$$
0<u_{y}(r)<v_{y}(r)<y, \quad \text { for every } r \in\left(0, R_{y}\right) ;
$$

and

$$
v_{y}(r) \downarrow 0, \text { as } \quad r \uparrow R_{y} .
$$

Indeed, by Lemma 3.3, $I_{0}$ is open; and so $y \notin I_{0}$. Therefore, $r_{y}=R_{y}$, and (3.6) follows.

Furthermore, since $y \notin I_{0}$, it follows from Proposition 3.2 that there exists $\left(s_{n}\right)_{n \in \mathbf{N}} \subset\left[0, R_{y}\right)$ such that $s_{n} \uparrow R_{y}$ and $v_{y}^{2}\left(s_{n}\right)-u_{y}^{2}\left(s_{n}\right) \rightarrow 0$, as $n \rightarrow \infty$. On the other hand, given $0 \leqq r \leqq R_{y}$, it follows from the fact that $y \in \overline{I_{0}}$, Lemma 3.6 and the continuous dependence of the solution on $x$ that $\left(u_{y}(r), v_{y}(r)\right) \in \Delta$. Note also that by Lemma $2.4, \Delta \cap\{u=v\}=\{(0,0)\}$. Therefore, we must have $v_{y}\left(s_{n}\right) \rightarrow 0$, as $n \rightarrow \infty$. Since $v_{y}$ is nonincreasing on $\left[0, R_{y}\right)$, it follows that $v_{y} \downarrow 0$, as $r \uparrow R_{y}$. Hence (3.7).

It follows that $\left(u_{y}, v_{y}\right)$ is an admissible positive solution, with radius $R_{y}$. This completes the proof.

\section{Further Results}

Let $(u, v)$ be an admissible positive solution of (1.4)-(1.7) of radius $R$, and let $x=v(0)$. Since $u>0$ on $(0, R)$, it follows in particular from Eq. (2.1) that $x>a$. Therefore, with the notation of Sect. 3, we must have $R=R_{x}=r_{x}$. Conversely, if $x>a$ is such that $r_{x}=R_{x}$, it is clear that $\left(u_{x}, v_{x}\right)$ is an admissible positive solution of (1.4)-(1.7) of radius $R_{x}$. Therefore, studying the properties of admissible positive solutions is equivalent to study the properties of $\left(u_{x}, v_{x}\right)$, where $x>a$ is such that $r_{x}=R_{x}$.

We begin with the following simple lemma.

Lemma 4.1. Assume that $\phi$ is bounded at the origin, and let $x>a$ be such that $r_{x}=R_{x}$. Then, $R_{x}=\infty$.

Proof. By Eq. (2.1), we have

$$
\left|v_{x}^{\prime}\right| \leqq C u_{x} \leqq C v_{x}, \quad \text { on }\left(0, R_{x}\right),
$$

with $C=\phi(0)+m+\omega$. It follows that

$$
v_{x}^{\prime}+C v_{x} \geqq 0 \text { on }\left(0, R_{x}\right) .
$$

Integrating the above equation, we obtain

$$
v_{x}(r) \geqq x e^{-c r}, \text { for } r \in\left(0, R_{x}\right) .
$$

Hence the result.

Next, we consider the case where $\phi$ is singular at the origin. Let us introduce the function $\psi$ as follows. Let $\Phi^{(-1)}$ be the inverse function of $\Phi$. Since $\Phi$ is increasing, positive, concave and $\Phi(0)=0$, it follows that $\Phi^{(-1)}$ is positive, 
increasing and convex, and that $\Phi^{(-1)}(0)=0$. Let

$$
\psi(s)=\phi\left(\Phi^{(-1)}(s)\right), \text { for } s>0 .
$$

It follows that $\psi$ is positive and nonincreasing, and that $\psi(0)=\phi(0)$.

Lemma 4.2. Assume that $\phi(0)=\infty$, and let $x>a$ be such that $R_{x}=r_{x}$. Then,

(i) $\frac{u(r)}{v(r)} \rightarrow 1$, as $r \uparrow R_{x}$;

(ii) for every $\varepsilon \in(0,1)$, there exists $\rho_{\varepsilon} \in\left(0, R_{x}\right)$ such that

$$
\psi\left(2\left(\omega-\frac{1}{R_{x}}\right)(1+\varepsilon) v_{x}^{2}\right) \leqq \phi\left(v_{x}^{2}-u_{x}^{2}\right) \leqq \psi\left(2\left(\omega-\frac{1}{R_{x}}\right)(1-\varepsilon) v_{x}^{2}\right),
$$

for all $r \in\left[\rho_{\varepsilon}, R_{x}\right)$.

Proof. By Lemma 3.5, $\tilde{H}_{1 /(\omega-\delta)}\left(u_{x}(r), v_{x}(r)\right) \leqq 0$, for every $r \in\left(\frac{1}{\omega-\delta}, r_{x}\right)$. It follows from Lemma 2.4 (i) that $\left(u_{x}, v_{x}\right)$ is between the curves $G_{1 /(\omega-\delta)}$ and the diagonal $\{u=v\}$, for $r \in\left(\frac{1}{\omega-\delta}, r_{x}\right)$. Therefore, (i) follows from Lemma 2.4 (iv), since $u_{x}+v_{x} \rightarrow 0$, as $r \rightarrow R_{x}$.

Let $0<\tau \leqq r<R_{x}$. We have

$$
\frac{u_{x}(r)}{r}-\frac{v_{x}(r)}{\tau}=\frac{u_{x}(r)-v_{x}(r)}{r}-v_{x}(r)\left(\frac{1}{\tau}-\frac{1}{r}\right) \leqq 0 .
$$

When Lemma 2.2 is applied, it follows that $\tilde{H}_{\tau}\left(u_{x}, v_{x}\right)$ is nondecreasing on $\left(\tau, R_{x}\right)$. Since furthermore $\widetilde{H}_{\tau}\left(u_{x}, v_{x}\right) \rightarrow 0$, as $r \uparrow R_{x}$, it follows that $\widetilde{H}_{\tau}\left(u_{x}, v_{x}\right) \leqq 0$, on $\left[\tau, R_{x}\right)$. This implies that

$$
\Phi\left(v_{x}^{2}(r)-u_{x}^{2}(r)\right)+(m+\omega)\left(v_{x}^{2}(r)-u_{x}^{2}(r)\right) \geqq 2\left(\omega-\frac{1}{r}\right) v_{x}^{2}(r),
$$

for all $r \in\left[\frac{1}{\omega}, R_{x}\right)$. Note that $\Phi(s) / s \rightarrow \infty$, as $s \downarrow 0$; and so,

$$
\frac{\Phi\left(v_{x}^{2}(r)-u_{x}^{2}(r)\right)+(m+\omega)\left(v_{x}^{2}(r)-u_{x}^{2}(r)\right)}{\Phi\left(v_{x}^{2}(r)-u_{x}^{2}(r)\right)} \downarrow 1, \quad \text { as } \quad r \uparrow R_{x} .
$$

Given $\varepsilon \in(0,1)$, it follows from (4.2)-(4.3) that there exists $\rho_{\varepsilon} \in\left(0, R_{x}\right)$ such that

$$
\Phi\left(v_{x}^{2}(r)-u_{x}^{2}(r)\right) \geqq 2\left(\omega-\frac{1}{R_{x}}\right)(1-\varepsilon) v_{x}^{2}(r), \quad \text { for } \quad r \in\left(\rho_{\varepsilon}, R_{x}\right) .
$$

The right-hand side inequality of (ii) follows by applying $\Phi^{(-1)}$, then $\phi$.

For proving the left-hand side inequality of (ii), we consider two cases. If $R_{x}=\infty$, we apply the inequality $H\left(u_{x}, v_{x}\right) \geqq 0$, on $(0, \infty)$. This implies that

$$
\Phi\left(v_{x}^{2}(r)-u_{x}^{2}(r)\right) \leqq-(m+\omega)\left(v_{x}^{2}(r)-u_{x}^{2}(r)\right)+2 \omega v_{x}^{2}(r) \leqq 2 \omega v_{x}^{2}(r),
$$

for all $r \in\left(0, R_{x}\right)$. The left-hand side inequality of (ii) follows by applying $\Phi^{(-1)}$, then $\phi$. 
If $R_{x}<\infty$, consider $\tau>R_{x}$. It follows from (i) that

$$
\frac{1}{v_{x}(r)}\left(\frac{u_{x}(r)}{r}-\frac{v_{x}(r)}{\tau}\right)=\frac{1}{r}\left(\frac{u_{x}(r)}{v_{x}(r)}-1\right)+\frac{1}{r}-\frac{1}{\tau} \underset{r \uparrow R_{x}}{\longrightarrow} \frac{1}{R_{x}}-\frac{1}{\tau}>0 .
$$

It follows that there exists $\theta \in\left(0, R_{x}\right)$ such that

$$
\frac{u_{x}(r)}{r}-\frac{v_{x}(r)}{\tau} \geqq 0, \quad \text { for } r \in\left[\theta, R_{x}\right) .
$$

By Lemma 2.2, this implies that $\tilde{H}_{\tau}\left(u_{x}, v_{x}\right)$ is nonincreasing on $\left(\theta, R_{x}\right)$. Since furthermore $\tilde{H}_{\tau}\left(u_{x}, v_{x}\right) \rightarrow 0$, as $r \uparrow R_{x}$. it follows that $\tilde{H}_{\tau}\left(u_{x}, v_{x}\right) \geqq 0$, on $\left[\theta, R_{x}\right)$. This means that

$$
\Phi\left(v_{x}^{2}(r)-u_{x}^{2}(r)\right) \leqq-(m+\omega)\left(v_{x}^{2}(r)-u_{x}^{2}(r)\right)+2\left(\omega-\frac{1}{\tau}\right) v_{x}^{2}(r) \leqq 2\left(\omega-\frac{1}{\tau}\right) v_{x}^{2}(r)
$$

for all $r \in\left(\theta, R_{x}\right)$. Given $\varepsilon \in(0,1)$, one can choose $\tau>R_{x}$ such that

$$
2\left(\omega-\frac{1}{\tau}\right) \leqq 2\left(\omega-\frac{1}{R_{x}}\right)(1+\varepsilon)
$$

and it follows from (4.4) that there exists $\rho_{\varepsilon} \in\left(0, R_{x}\right)$ such that

$$
\Phi\left(v_{x}^{2}(r)-u_{x}^{2}(r)\right) \leqq 2\left(\omega-\frac{1}{R_{x}}\right)(1+\varepsilon) v_{x}^{2}(r), \text { for } r \in\left(\rho_{\varepsilon}, R_{x}\right)
$$

The left-hand side inequality of (ii) follows by applying $\Phi^{(-1)}$, then $\phi$. This completes the proof.

Proof of Theorem 1.3. (i) Consider $x>a$ such that $R_{x}=r_{x}$. It follows from Proposition 2.7 (i) that

$$
R_{x}>\frac{1}{\omega-\delta}>\frac{1}{\omega}
$$

Hence (i).

(ii) Note that the assumption

$$
\int_{0}^{1} \frac{d s}{G(s)}<\infty
$$

is equivalent to

$$
\int_{0}^{1} \frac{d s}{\Phi(s)}<\infty
$$

Furthermore, putting $v=\Phi(s)$, we have

$$
\int_{0}^{1} \frac{d s}{G(s)}=\int_{0}^{\Phi(1)} \frac{d v}{v \Phi^{\prime}\left(\Phi^{(-1)}(v)\right)}=\int_{0}^{\Phi(1)} \frac{d v}{v \psi(v)},
$$

where $\psi$ is defined by (4.1). Therefore, assumption (4.5) is equivalent to

$$
\int_{0}^{1} \frac{d s}{s \psi(s)}<\infty
$$


So, suppose that (4.6) holds. In particular, $\phi$ must be unbounded at the origin; and so we can apply Lemma 4.2. Consider $x>a$ such that $R_{x}=r_{x}$. It follows from (2.2), and Lemma 4.2 that there exists $\rho \in\left(0, R_{x}\right)$ such that

$$
\begin{aligned}
& 0=v_{x}^{\prime}+u_{x}\left(\phi\left(v_{x}^{2}-u_{x}^{2}\right)+\omega+m\right) \geqq v_{x}^{\prime}+\frac{1}{2} v_{x}\left(\phi\left(v_{x}^{2}-u_{x}^{2}\right)+\omega+m\right) \\
& \geqq v_{x}^{\prime}+\frac{1}{2} v_{x} \phi\left(v_{x}^{2}-u_{x}^{2}\right) \geqq v_{x}^{\prime}+\frac{1}{2} v_{x} \psi\left(4\left(\omega-\frac{1}{R_{x}}\right) v_{x}^{2}\right),
\end{aligned}
$$

on $\left(\rho, R_{x}\right)$. It follows that for some $A>0$,

$$
2 \int_{\rho}^{R_{x}} \frac{v_{x}^{\prime}(r) d r}{v_{x}(r) \varphi\left(A v_{x}(r)^{2}\right)}+R_{x}-\rho \leqq 0
$$

This means that

$$
R_{x} \leqq \rho+\int_{0}^{v_{x}(\rho)} \frac{d s}{s \psi\left(A s^{2}\right)}=\rho+\frac{1}{2} \int_{0}^{A v_{x}(\rho)^{2}} \frac{d s}{s \psi(s)}<\infty
$$

and so, $R_{x}<\infty$.

Conversely, assume that $x$ is as above and that $R_{x}$ is finite. In particular, $\phi$ is singular at the origin, by Lemma 4.1; and so, we can apply Lemma 4.2. It follows from (2.2) and Lemma 4.2 that there exists $\rho \in\left(0, R_{x}\right)$ such that

$$
\begin{aligned}
0 & =v_{x}^{\prime}+u_{x}\left(\phi\left(v_{x}^{2}-u_{x}^{2}\right)+\omega+m\right) \leqq v_{x}^{\prime}+v_{x}\left(\phi\left(v_{x}^{2}-u_{x}^{2}\right)+\omega+m\right) \\
& \leqq v_{x}^{\prime}+v_{x}\left(\left(\psi\left(\omega-\frac{1}{R_{x}}\right) v_{x}^{2}\right)+\omega+m\right) \leqq v_{x}^{\prime}+v_{x}\left(\psi\left(\omega v_{x}^{2}\right)+\omega+m\right),
\end{aligned}
$$

on $\left(\rho, R_{x}\right)$. Since $\phi(0)=\psi(0)=\infty$, it follows that, by possibly choosing $\rho$ closer to $\boldsymbol{R}_{x}$,

$$
0 \leqq v_{x}^{\prime}+2 v_{x} \psi\left(\omega v_{x}^{2}\right)
$$

on $\left(\rho, R_{x}\right)$. Proceeding as above, we obtain

$$
\int_{0}^{\omega v_{x}(\rho)^{2}} \frac{d s}{s \psi(s)} \leqq 4\left(R_{x}-\rho\right)<\infty ;
$$

and so, (4.6) holds. This completes the proof.

Proof of Theorem 1.4. It is sufficient to show that if $x>a$ is such that $R_{x}=r_{x}=\infty$, then $v_{x}$ (hence $u_{x}$ ) has an exponential decay as $r \rightarrow \infty$, and $v_{x}^{\prime}, v_{x}^{\prime} \in L^{1}(0, \infty)$. Indeed, it follows from (2.1)-(2.2) that

$$
\left(u_{x}+v_{x}\right)^{\prime}+\left(u_{x}+v_{x}\right)\left(\phi\left(v_{x}^{2}-u_{x}^{2}\right)-\omega+m\right)=-\frac{2 u_{x}}{r}-2 \omega u_{x} \leqq 0 .
$$

Since $v_{x}^{2}-u_{x}^{2} \rightarrow 0$, as $r \rightarrow \infty$, it follows from Remark 2.1 (iii) that there exists $\delta>0$ such that

$$
\left(u_{x}+v_{x}\right)^{\prime}+\delta\left(u_{x}+v_{x}\right) \leqq 0, \quad \text { for } r \text { large. }
$$

The exponential decay follows easily. Note also that by (2.1)-(2.2) and Remark 2.1 (iii), there exists $r_{0}$ such that

$$
u_{x}^{\prime}<0 \text { an } v_{x}^{\prime}<0, \text { for } r \geqq r_{0} .
$$


Since both $u_{x}$ and $v_{x}$ converge to 0 , as $r \rightarrow \infty$, it follows that $v_{x}^{\prime}, v_{x}^{\prime} \in L^{1}\left(r_{0}, \infty\right)$. Since $u_{x}^{\prime}$ and $v_{x}^{\prime}$ are bounded on $\left[0, r_{0}\right]$, the result follows.

Finally, it remains to prove Theorem 1.6. It will be a consequence of the following two lemmas.

Lemma 4.3. Let $x>a$ be such that $R_{x}=r_{x}<\infty$. Define the functions $\hat{u}, \hat{v}, h, k$ by

$$
\hat{u}(r)=\left\{\begin{array}{ll}
u_{x}(r), & \text { if } 0 \leqq r<R_{x} ; \\
0, & \text { if } r>R_{x} ;
\end{array} \quad \hat{v}(r)=\left\{\begin{array}{lll}
v_{x}(r), & \text { if } 0 \leqq r<R_{x} \\
0, & \text { if } r>R_{x} ;
\end{array}\right.\right.
$$

and

$$
\begin{gathered}
h(r)=\left\{\begin{array}{l}
-2 \frac{u_{x}}{r}-v_{x}(r)\left(\phi\left(v_{x}^{2}-u_{x}^{2}\right)-\omega+m\right), \text { if } 0 \leqq r<R_{x} ; \\
0, \text { if } \quad r>R_{x} ;
\end{array}\right. \\
k(r)= \begin{cases}-u_{x}(r)\left(\phi\left(v_{x}^{2}-u_{x}^{2}\right)+\omega+m\right), & \text { if } 0 \leqq r<R_{x} ; \\
0, & \text { if } \quad r>R_{x} .\end{cases}
\end{gathered}
$$

Then, $\hat{u}, \hat{v} \in W^{1,1}(0, \infty), h, k \in L^{1}(0, \infty)$, and we have

in $\mathscr{D}^{\prime}(0, \infty)$.

$$
\left\{\begin{array}{l}
\hat{u}^{\prime}=h \\
\hat{v}^{\prime}=k
\end{array}\right.
$$

Proof. Note that $\hat{u}, \hat{v} \in C^{1}\left(\left[0, R_{x}\right)\right), h, k \in C\left(\left[0, R_{x}\right)\right)$, and that by (2.1)-(2.2),

$$
\left\{\begin{array}{l}
\hat{u}^{\prime}=h, \\
\hat{v}^{\prime}=k,
\end{array}\right.
$$

on $\left[0, R_{x}\right)$. Evidently, we also have $\hat{u}, \hat{v} \in C^{1}\left(R_{x}, \infty\right), h, k \in C\left(R_{x}, \infty\right)$ and

$$
\left\{\begin{array}{l}
\hat{u}^{\prime}=h, \\
\hat{v}^{\prime}=k,
\end{array}\right.
$$

on $\left(R_{x}, \infty\right)$. Note also that $\hat{u}, \hat{v}$ are continuous on $[0, \infty)$. Therefore, the result follows if we show that $h, k$ are integrable near $R_{x}$. In other words, we have to show that

$$
\int_{0}^{R_{x}}\left(\left|u_{x}^{\prime}\right|+\left|v_{x}^{\prime}\right|\right) d r<\infty
$$

Since $u_{x}$ and $v_{x}$ converge to 0 , as $r \uparrow R_{x}$, it follows from (2.1)-(2.2) and Remark 2.1 (iii) that there exists $\rho \in\left(0, R_{x}\right)$ such that $u_{x}^{\prime}<0$ and $v_{x}^{\prime}<0$, on $\left(\rho, R_{x}\right)$. It follows that

$$
\int_{\rho}^{R_{x}}\left(\left|u_{x}^{\prime}\right|+\left|v_{x}^{\prime}\right|\right) d r=-\int_{\rho}^{R_{x}}\left(u_{x}^{\prime}+v_{x}^{\prime}\right) d r=u_{x}(\rho)+v_{x}(\rho)<\infty .
$$

Since $u_{x}^{\prime}$ and $v_{x}^{\prime}$ are bounded on $(0, \rho),(4.7)$ follows. Hence the result.

Lemma 4.4. Let $x>a$ be such that $R_{x}=r_{x}<\infty$. Define the function $\lambda$ by

$$
\lambda(s)=\frac{d}{d s}(\Phi(s))^{3 / 2}, \quad \text { for } \quad s>0 .
$$


Then,

$$
\underset{r \uparrow R_{x}}{\liminf } u_{x}^{\prime}(r)=\liminf _{r \uparrow R_{x}} v_{x}^{\prime}(r)=-c \underset{s \downarrow 0}{\lim \sup } \lambda(s),
$$

and

$$
\underset{r \uparrow R_{x}}{\lim \sup } u_{x}^{\prime}(r)=\underset{r \uparrow R_{x}}{\lim \sup } v_{x}^{\prime}(r)=-c \liminf _{s \downarrow 0} \lambda(s),
$$

where $c=\frac{2}{3 \sqrt{\alpha}}$ and $\alpha=2\left(\omega-\frac{1}{R_{x}}\right)$. In particular, $\lambda$ has the (finite or infinite) limit $l$ as $s \downarrow 0$, if and only if both $u_{x}^{\prime}$ and $v_{x}^{\prime}$ have the limit $-c l$, as $r \uparrow R_{x}$.

Proof. Note first that

$$
\lambda(s)=\frac{3}{2} \sqrt{\Phi(s)} \phi(s) .
$$

Given $a>0$, and putting $a x^{2}=\Phi(s)$, it follows that

$$
\lambda(s)=\frac{3}{2} \sqrt{a x} \psi\left(a x^{2}\right)
$$

and so,

$$
\left\{\begin{array}{l}
\liminf _{x \downarrow 0} x \psi\left(a x^{2}\right)=\frac{3}{2 \sqrt{a}} \underset{s \downarrow 0}{\liminf } \lambda(s), \\
\underset{x \downarrow 0}{\lim \sup } x \psi\left(a x^{2}\right)=\frac{3}{2 \sqrt{a}} \limsup _{s \downarrow 0} \lambda(s) .
\end{array}\right.
$$

Note also that by Lemma $4.1, \phi$ is singular at the origin. Therefore, it follows from Lemma 4.2 that, given $\varepsilon \in(0,1)$, we have

$$
v_{x} \phi\left(v_{x}^{2}-u_{x}^{2}\right) \leqq v_{x} \psi\left((1-\varepsilon) \alpha v_{x}^{2}\right)
$$

on $\left(\rho_{\varepsilon}, R_{x}\right)$; and so, by (2.1) and (4.10),

$$
\underset{r \uparrow R_{x}}{\lim \sup }\left(-u_{x}^{\prime}\right) \leqq \underset{x \downarrow 0}{\limsup } x \psi\left((1-\varepsilon) \alpha x^{2}\right)=\frac{3}{2 \sqrt{(1-\varepsilon) \alpha}} \limsup _{s \downarrow 0} \lambda(s) .
$$

Making $\varepsilon \downarrow 0$, we obtain

$$
\underset{r \uparrow R_{x}}{\lim \sup }\left(-u_{x}^{\prime}\right) \leqq c \limsup _{s \downarrow 0} \lambda(s) .
$$

Furthermore, applying again Lemma 4.2, we obtain

$$
v_{x} \phi\left(v_{x}^{2}-u_{x}^{2}\right) \geqq v_{x} \psi\left((1+\varepsilon) \alpha v_{x}^{2}\right),
$$

on $\left(\rho_{\varepsilon}, R_{x}\right)$; and so, by (2.1) and (4.10),

$$
\underset{r \uparrow R_{x}}{\lim \sup }\left(-u_{x}^{\prime}\right) \geqq \underset{x \downarrow 0}{\limsup } x \psi\left((1+\varepsilon) \alpha x^{2}\right)=\frac{3}{2 \sqrt{(1+\varepsilon) \alpha}} \limsup _{s \downarrow 0} \lambda(s) .
$$

Making $\varepsilon \downarrow 0$, we obtain

$$
\underset{r \uparrow R_{x}}{\lim \sup }\left(-u_{x}^{\prime}\right) \geqq c \underset{s \downarrow 0}{\limsup } \lambda(s) .
$$


It follows that

$$
\underset{r \uparrow R_{x}}{\lim \sup }\left(-u_{x}^{\prime}\right)=c \underset{s \downarrow 0}{\lim \sup } \lambda(s) .
$$

Clearly, the same holds for the lim inf, and working with Eq. (2.2), one obtains the same identities for $v_{x}^{\prime}$. Hence the result.

Remark 4.5. Note that $\phi(s)=s^{-p}$, for $0<p<1$, verifies the assumptions of Theorem 1.2. In addition, we have

$$
\int_{0}^{1} \frac{d s}{\Phi(s)}<\infty
$$

Therefore, all admissible solutions have compact support. Furthermore, it follows from Lemma 4.4 that if $(u, v)$ is an admissible solution of radius $R$, then for $0<p<1 / 3,\left|u^{\prime}\right|+\left|v^{\prime}\right| \rightarrow 0$, as $r \uparrow R$. In particular, $\hat{u}, \hat{v} \in C^{1}([0, \infty))$. For $p=1 / 3$, both $u^{\prime}$ and $v^{\prime}$ converge to the same finite negative limit, as $r \uparrow R$. For $p>1 / 3$, both $u^{\prime}$ and $v^{\prime}$ converge to $-\infty$, as $r \uparrow R$.

Proof of Theorem 1.6. The first part of the statement follows easily from Lemma 4.3 and formulas (1.11) and (1.12), and the second part follows from Lemma 4.4.

Acknowledgements. This work was partially supported by the "Action Intégrée Franco-Espagnole" \#88/077, and concerning one of the authors (L. Vázquez) by the U.S.-Spain Joint Committee for Scientific and Technological cooperation under grant CCB-8509/001.

\section{References}

1. Balabane, M.: États excités pour une équation de Dirac non linéaire. Méthode des "coefficients gelés." École Polytechnique, Centre de Mathématiques. Exposé \#XXIV: 1988

2. Balabane, M., Cazenave, T., Merle, F., Douady, A.: Existence of excited states for a nonlinear Dirac field. Commun. Math. Phys. 119, 153-176 (1988)

3. Cazenave, T.: On the existence of stationary states for classical nonlinear Dirac fields. In: Hyperbolic Systems and Mathematical Physics. Textos e Notas \#41, C.M.A.F., Lisbon, 1989 61-114

4. Cazenave, T., Vázquez, L.: Existence of localized solutions for a classical nonlinear Dirac field. Commun. Math. Phys. 105, 35-47 (1986)

5. Chodos, A., Jaffe, R. L., Johnson, K., Thorn, C. B., Weisskopf, V. F.: Mew extended model of hadrons. Phys. Rev. D9, 3471-3495 (1974)

6. Finkelstein, R., Fronsdal, C. F., Kaus, P.: Nonlinear spinor fields. Phys. Rev. 103, 1571-1579 (1956)

7. Heisenberg, W.: Doubts and hopes in quantumelectrohydrodynamics. Physica 19, 897-908 (1953)

8. Ivanenko, D.: Sov. Phys. 13, 141-149 (1938)

9. Johnson, K.: A field theory Lagrangian for the MIT bag model. Phys. Lett. 78B, 259-262(1978)

10. Mathieu, P.: New Lagrangian formalism for the bag. Phys. Rev. D31, 2145-2147 (1985)

11. Mathieu, P., Saly, R.: Baglike solutions of a Dirac equation with fractional nonlinearity. Phys. Rev. D29, 2879-2883 (1984)

12. Merle, F.: Existence of stationary states for nonlinear Dirac equations. J. Diff. Eq. 74, 50-68 (1988)

13. Rañada, A. F.: Classical nonlinear Dirac field models of extended particles. In: Quantum theory, groups, fields and particles. Barut, A. O. (ed.) Amsterdam: Reidel 1983 
14. Soler, M.: Classical, stable, nonlinear spinor field with positive rest energy. Phys. Rev. D1, 2766-2769 (1970)

15. Soler, M.: Classical electrodynamics for a nonlinear spinor field: Perturbative and exact approaches. Phys. Rev. D8, 3424-3429 (1973)

16. Stubbe, $\mathrm{J}$ :: Constrained minimization problems in Orlicz spaces with applications to minimum action solutions of nonlinear scalar field equations in $\mathbf{R}^{n}$. Preprint, BI TP $86 / 10$, Bielefeld University: 1986

17. Vázquez, L.: Localized solutions of a nonlinear spinor field. J. Phys. A10, 1361-1368 (1977)

18. Wakano, M.: Intensely localized solutions of the classical Dirac-Maxwell field equations. Prog. Theor. Phys. (Kyoto) 35, 1117-1141 (1966)

19. Weyl, H.: A remark on the coupling of gravitation and electron. Phys. Rev. 77, 699-701 (1950)

Communicated by A. Jaffe 